\title{
Model-Based Recognition of 3D Objects from One View
}

\author{
Isaac Weiss ${ }^{\star}$ and Manjit Ray \\ Center for Automation Research, University of Maryland, \\ College Park, MD 20742-3275, USA \\ weiss@cfar.umd.edu
}

\begin{abstract}
In this work we treat major problems of object recognition which have previously received little attention. Among them are the loss of depth information in the projection from $3 \mathrm{D}$ to $2 \mathrm{D}$, and the complexity of finding feature correspondences in general cases. This treatment enables us to recognize objects in difficult real-world situations.

It is well known that there are no geometric invariants of a projection from $3 \mathrm{D}$ to $2 \mathrm{D}$. However, given some modeling assumptions about the $3 \mathrm{D}$ object, such invariants can be found. The modeling assumptions can be either a particular model or a generic assumption about a class of models. Here we deal with both situations. We find invariant connections between a 2D image and a 3D model under general projective projection. We give a geometric interpretation of the method as an invariant model in 3D invariant space, illuminated by invariant light rays, converging to an invariant camera center in the same space. We demonstrate the method on real images.

This work differs from related work in the following ways: 1) Most work in invariants has concentrated on transformations of the same dimensionality, mainly 2D to $2 \mathrm{D}$ projections. We deal here with the problem of projecting a 3D object onto a $2 \mathrm{D}$ image, which is of greater relevance to object recognition. 2) Much of the previous work is done on multiple images, such as the work on camera calibration. This usually requires knowledge of the correspondence between images. We concentrate on single images, but we also apply our method to finding correspondences in multiple images without prior knowledge or expensive search.
\end{abstract}

Keywords: object recognition, model based vision, invariants

\section{Introduction}

Almost all the work on invariants so far has been concerned with transformations between spaces of equal dimensionality, e.g. [31,32,19]. In the single-view case, invariants were found for the projection of a planar shape onto the image,

\footnotetext{
* The author is grateful for the support of the Air Force Office of Scientific Research under grant F49620-92-J-0332, the Defense Advanced Research Projects Agency (DARPA Order No. E655) and Air Force Wright Laboratory under Grant F4962096-1-0355, and the Office of Naval Research under Grant N00014-95-1-0521.
} 
although the planar shape was embedded in 3D. For real 3D objects, most of the work has involved multiple views with known correspondence, which amounts to a $3 \mathrm{D}$ to $3 \mathrm{D}$ projection. Yet, humans have little problem recognizing a $3 \mathrm{D}$ object from a single $2 \mathrm{D}$ image.

This recognition ability cannot be based on pure geometry, since it has been shown (e.g. [6]) that there are no geometric invariants of a projection from 3D to $2 \mathrm{D}$. Thus, when we only have $2 \mathrm{D}$ geometric information, we need to use some modeling assumptions to recover the 3D shape.

There are several possibilities for a modelling assumption. The simplest one is having a library of specific $3 \mathrm{D}$ models. In theory there could be many models that project into the same image, so an object cannot be identified uniquely. In practice, however, in most cases there is only one or very few models in the database that would project to the same image, so it is possible to recognize them.

Another possibility is to have more generic assumptions, rather than specific models. One such assumption can be that the visible object is symmetric in 3D. More general assuptions for curved objects were studied in [36]. In this paper we deal mainly with the two assumptions mentioned above, namely specific models and symmetry. To a lesser extent we use other assumptions, such as that a vanishing point in a $2 \mathrm{D}$ image indicates parallel lines in $3 \mathrm{D}$.

The outline of our recognition method is as follows. We define a 3D invariant space, namely a space with three invariant coordinates $I_{1}, I_{2}, I_{3}$. Given a $3 \mathrm{D}$ model, we can extract a set of such invariant triplets from it, so it can be represented as a set of points in the invariant space. Given an image of the model, the depth information is lost so the invariant point cannot be recovered. However, we show that we can draw a set of "invariant light rays" in 3D, each ray passing through a 3D invariant model point (Fig. 1). When enough rays intersect the model points in 3D, we can safely assume that the model is indeed the one visible in the image. We do not need to search for correspondences. We can also see that the rays converge at a point in the invariant space that represents the location of the camera center with respect to the model. Thus it is easy to find the pose of the model. Given this, we can project the original (non-invariant) model onto the image. That makes it possible to perform a more exact match between the model projection and the given image of the object.

In summary, the invariant modeling assumption and the object descriptors make it possible to perform recognition regardless of viewpoint and with no need for a search for correspondence.

The use of modeling for shape recovery from single images is of course not new. However, most of the earlier work was not concerned with viewpoint invariance. Some recent research does use invariance in modeling. However, most of it uses very specific modeling assumptions that cannot be applied to general shapes. A major example is the assumption that the objects are composed of "general cylinders" $[4,37]$ of various forms. The invariance and generality of this assumption are limited. A subset of this is the assumption that the object is a surface of revolution [38]. Another assumption is that various corners visible in 
the image are right-angled in 3D [11]. Yet another approach is to assume that it is sufficient to characterize an object by special local properties such as tangencies and inflection points [30]. Of course this ignores the information available between the inflection points.

The above examples represent only a small part of the quite active research on invariants. However, unlike our interest here, most of the recent activity is concerned not with single images but with multiple images. These methods can recover the 3D geometry without modeling. However, they require knowledge of the correspondence between features in the different images. Correspondence is a difficult and generally unsolved problem, leading to a high-dimensional search space. Among such methods are those using the trilinear tensor $[22,2,18]$. They require finding a substantial number of corresponding points and lines in at least three images. This can be accomplished only for very small disparities. Much of the multiple image work is intended for camera calibration, e.g $[9,17,29]$, rather than object recognition. Techniques for multiple images have been applied to a single image, when "repetitive" or symmetric structures can be found in it $[8,38,23]$. These parts can be viewed as separate images, but the correspondence between their features still needs to be found. We can deal with both symmetric and non-symmetric objects in a single image, without correspondence.

Work that directly connects 2D and 3D quantitative invariants was done in [12] and [26]. That work comsidered affine point-sets only, while here we derive the projective case as well. They did not use modeling assumptions, and therefore a 3D shape could not be recognized uniquely.

There is also considerable research on curves. Differential projective geometry was used for plane curves in e.g. [33,19,34,5,20,28]. More specialized approaches, including the use of pairs of co-planar conics, can be found in $[25,27,3,21,16,13]$. More general 3D curves were studied in [36].

\section{Point Set Invariants}

\subsection{General Dependencies among Invariants}

Here we describe our method of connecting 3D and 2D invariants by applying it to point sets. We rederive all the results in [26] in a much simpler way, using elementary algebra rather than algebraic geometry. We then add the new projective case.

We denote 3D world coordinates by $\mathbf{X}$, and $2 \mathrm{D}$ image coordinates by $\mathbf{x}$. We have five points $\mathbf{X}_{i}, i=1, \ldots, 5$ in 3D space, of which at least the first four are not coplanar. They are projected into $\mathbf{x}_{i}$ in the image. The correspondence is assumed to be known for now. In a 3D projective or affine space, five points cannot be linearly independent. We can express the fifth point as a linear combination of the first four:

$$
\mathbf{X}_{5}=a \mathbf{X}_{1}+b \mathbf{X}_{2}+c \mathbf{X}_{3}+d \mathbf{X}_{4}
$$

In the projective case the coefficients $a, b, c, d$ are not uniquely determined because the point coordinates can be multiplied by an arbitrary factor. In the affine 
case, the coefficients are constrained by the requirement that the fourth homogeneous coordinate is always 1 , again leaving only three independent coefficients. Because the projection from 3D to 2D is linear (in homogeneous coordinates), the same dependence holds in 2D:

$$
\mathbf{x}_{5}=a \mathbf{x}_{1}+b \mathbf{x}_{2}+c \mathbf{x}_{3}+d \mathbf{x}_{4}
$$

Since determinants are relative invariants of a projective or affine transformation, we look at the determinants formed by these points in both 3D and 2D. Any four of the five points in 3D, expressed in four homogeneous coordinates, can form a determinant $M_{i}$. We can give the determinant the same index as the fifth point that was left out. For example,

$$
M_{1}=\left|\mathbf{X}_{2}, \mathbf{X}_{3}, \mathbf{X}_{4}, \mathbf{X}_{5}\right|
$$

Similarly, in the 2D projection, any three of the five points can form a determinant $m_{i j}$, with indices equal to those of the points that were left out, e.g.

$$
m_{12}=\left|\mathbf{x}_{3}, \mathbf{x}_{4}, \mathbf{x}_{5}\right|
$$

Since the points are not independent, neither are the determinants. Substituting the linear dependence (1) in $M_{1}$ above we obtain

$M_{1}=a\left|\mathbf{X}_{2}, \mathbf{X}_{3}, \mathbf{X}_{4}, \mathbf{X}_{1}\right|+b\left|\mathbf{X}_{2}, \mathbf{X}_{3}, \mathbf{X}_{4}, \mathbf{X}_{2}\right|+c\left|\mathbf{X}_{2}, \mathbf{X}_{3}, \mathbf{X}_{4}, \mathbf{X}_{3}\right|+d\left|\mathbf{X}_{2}, \mathbf{X}_{3}, \mathbf{X}_{4}, \mathbf{X}_{4}\right|$

As is well known, a determinant with two equal columns vanishes. Also, when columns are interchanged in a determinant, the sign of the determinant is reversed. Therefore we obtain

$$
M_{1}=a\left|\mathbf{X}_{2}, \mathbf{X}_{3}, \mathbf{X}_{4}, \mathbf{X}_{1}\right|=-a\left|\mathbf{X}_{1}, \mathbf{X}_{2}, \mathbf{X}_{3}, \mathbf{X}_{4}\right|=-a M_{5}
$$

Similarly for the other determinants, with a simplified notation:

$$
\begin{gathered}
M_{2}=|1,3,4,5|=b|1,3,4,2|=b|1,2,3,4|=b M_{5} \\
M_{3}=|1,2,4,5|=c|1,2,4,3|=-c|1,2,3,4|=-c M_{5} \\
M_{4}=|1,2,3,5|=d|1,2,3,4|=d M_{5}
\end{gathered}
$$

The coefficients $a, b, c, d$ can now be expressed as invariants, using the above relations:

$$
a=-\frac{M_{1}}{M_{5}}, \quad b=\frac{M_{2}}{M_{5}}, \quad c=-\frac{M_{3}}{M_{5}}, \quad d=\frac{M_{4}}{M_{5}}
$$

This is the standard solution for $a, b, c, d$ as unknowns in eq. (1).

Similar relations hold in the $2 \mathrm{D}$ projection:

$$
\begin{gathered}
m_{12}=|3,4,5|=a|3,4,1|+b|3,4,2|=a|1,3,4|+b|2,3,4|=a m_{25}+b m_{15} \\
m_{13}=|2,4,5|=a|2,4,1|+c|2,4,3|=a m_{35}-c m_{15} \\
m_{14}=|2,3,5|=a|2,3,1|+d|2,3,4|=a m_{45}+d m_{15}
\end{gathered}
$$

Other relations are linearly dependent on these. 


\subsection{Relation between 3D and 2D Invariants - Affine Case}

In the affine case the coefficients $a, b, c, d$ are absolute invariants. Therefore we can substitute the $a, b, c, d$ found in $3 \mathrm{D}$, eq. (2), directly into the $2 \mathrm{D}$ equations above. We obtain three relations between the 3D and the 2D invariants:

$$
\begin{aligned}
& M_{5} m_{12}+M_{1} m_{25}-M_{2} m_{15}=0 \\
& M_{5} m_{13}+M_{1} m_{35}-M_{3} m_{15}=0 \\
& M_{5} m_{14}+M_{1} m_{45}-M_{4} m_{15}=0
\end{aligned}
$$

These relations are obviously invariant to any affine transformation in both $3 \mathrm{D}$ and 2D. A 3D transformation will merely multiply all the $M_{i}$ by the same constant factor, which drops out of the equations. A 2D affine transformation multiplies all the $m_{i j}$ by the same constant factor, which again drops out. However, in the projective case each point can be independently multiplied by an arbitrary factor $\lambda_{i}$, which does not in general drop out. Thus the above relation is affine but not projective invariant.

The above relations become linearly dependent so that only two of them are meaningful. To see this, we first note a relationship between the $M_{i}$ which exists only in the affine case. We can write a determinant involving all five points as

$$
\left|\begin{array}{ccccc}
x_{1} & x_{2} & x_{3} & x_{4} & x_{5} \\
y_{1} & y_{2} & y_{3} & y_{4} & y_{5} \\
z_{1} & z_{2} & z_{3} & z_{4} & z_{5} \\
1 & 1 & 1 & 1 & 1 \\
1 & 1 & 1 & 1 & 1
\end{array}\right|=0
$$

The $M_{i}$ are minors of this determinant so we can write the above equation as

$$
M_{1}-M_{2}+M_{3}-M_{4}+M_{5}=0
$$

This is equivalent to writing $a+b+c+d=1$ in eq. (1), which ensures that the last coordinate equals 1 .

Similar relations can be derived in $2 \mathrm{D}$. We have

$$
\left|\begin{array}{cccc}
x_{2} & x_{3} & x_{4} & x_{5} \\
y_{2} & y_{3} & y_{4} & y_{5} \\
1 & 1 & 1 & 1 \\
1 & 1 & 1 & 1
\end{array}\right|=0
$$

leading to the relation

$$
m_{12}-m_{13}+m_{14}-m_{15}=0
$$

Similarly, from the determinant involving points $1,2,3,4$ we obtain the relation

$$
m_{15}-m_{25}+m_{35}-m_{45}=0
$$


We now look at the following linear combination of the invariant relations, eqs. $(6),(7),(8)$ :

$(6)-(7)+(8)=M_{5}\left(m_{12}-m_{13}+m_{14}\right)+M_{1}\left(m_{25}-m_{35}+m_{45}\right)+\left(-M_{2}+M_{3}-M_{4}\right) m_{15}=0$

Using the two relations above between the $m_{i j}$ we obtain

$$
m_{15}\left(M_{1}-M_{2}+M_{3}-M_{4}+M_{5}\right)=0
$$

which is an identity, due the the relation between the $M_{i}$ above. Thus, only two invariant relations, say (6),(7), are independent.

Similar results for this particular case can be obtained using Grassmannians, Schubert cycles and wedge-products [26]. These methods are hard to extend much beyond this point.

\subsection{Relations between 3D and 2D Absolute Affine Invariants}

It is easy now to derive the relation between the $3 \mathrm{D}$ and $2 \mathrm{D}$ absolute invariants. We define the 3D absolute invariants

$$
I_{1}=\frac{M_{1}}{M_{5}}, \quad I_{2}=\frac{M_{2}}{M_{5}}, \quad I_{3}=\frac{M_{3}}{M_{5}}
$$

and the $2 \mathrm{D}$ absolute invariants

$$
i_{12}=\frac{m_{12}}{m_{15}}, \quad i_{13}=\frac{m_{13}}{m_{15}}, \quad i_{25}=\frac{m_{25}}{m_{15}}, \quad i_{35}=\frac{m_{35}}{m_{15}}
$$

and obtain the following theorem:

Theorem 1. Given five points $\mathbf{X}_{i}$ in 3D, at least four of which are non-coplanar, the relation between their $3 D$ and $2 D$ absolute affine invariants is given by

$$
\begin{aligned}
& i_{12}+I_{1} i_{25}-I_{2}=0 \\
& i_{13}+I_{1} i_{35}-I_{3}=0
\end{aligned}
$$

Proof: Divide eqs. (6),(7) by $M_{5}$ and $m_{15}$ (assuming these do not vanish).

Given the $2 \mathrm{D}$ projection of a five-point set, we have thus obtained two equations for the three unknown $3 \mathrm{D}$ invariants $I_{1}, I_{2}, I_{3}$. Since all three invariants are needed to recover the five points in $3 \mathrm{D}$ (up to an affine transformation), we can recover the $3 \mathrm{D}$ quintuple only up to one free parameter.

A geometric interpretation and applications are described in the next section.

\subsection{Points and Directions}

Rather than dealing with a point, we can deal with a direction, namely a unit vector in $3 \mathrm{D}$ pointing in a certain direction. This can represent a direction of a major axis of the model or a direction of the camera axis. 
A direction is equivalent to a point on the infinite plane and can be written as $(x, y, z, 0)$, i.e. with a vanishing fourth coordinate. It will remain at infinity under affine transformation. Thus, the derivation leading to eqs. (6),(7),(8) remains valid. However, eq. (9) needs to be modified. For a direction $\mathbf{X}_{4}$ we can write the determinant

$$
\left|\begin{array}{ccccc}
x_{1} & x_{2} & x_{3} & x_{4} & x_{5} \\
y_{1} & y_{2} & y_{3} & y_{4} & y_{5} \\
z_{1} & z_{2} & z_{3} & z_{4} & z_{5} \\
1 & 1 & 1 & 0 & 1 \\
1 & 1 & 1 & 0 & 1
\end{array}\right|=0
$$

in which $M_{i}$ are the minors and we immediately obtain

$$
M_{1}-M_{2}+M_{3}+M_{5}=0
$$

The constraints on $m_{i j}$ also change, but it is easy to show that we again obtain a linear dependency among eqs. (6),(7),(8). Thus, Theorem 1 is still valid. A direction vector can be multiplied by an arbitrary constant, but $\mathbf{X}_{\mathbf{4}}, \mathbf{x}_{4}$ are common to all terms in the equations and thus their factors drop out.

\subsection{The Projective Case}

Our method is easily extended to the projective case, yielding new simple results such as Theorem 2 below. This case is more difficult because in "real" (Cartesian) coordinates the projective transformation is non-linear, causing other approaches $[1,15]$ to be quite cumbersome.

We start with the $3 \mathrm{D}$ quantities. To obtain invariants we need at least six points, having three projective invariants. We now have two linear dependencies rather than one:

$$
\begin{gathered}
\lambda_{5} \mathbf{X}_{5}=a \lambda_{1} \mathbf{X}_{1}+b \lambda_{2} \mathbf{X}_{2}+c \lambda_{3} \mathbf{X}_{3}+d \lambda_{4} \mathbf{X}_{4} \\
\lambda_{6} \mathbf{X}_{6}=a^{\prime} \lambda_{1} \mathbf{X}_{1}+b^{\prime} \lambda_{2} \mathbf{X}_{2}+c^{\prime} \lambda_{3} \mathbf{X}_{3}+d^{\prime} \lambda_{4} \mathbf{X}_{4}
\end{gathered}
$$

with $\lambda_{i}$ being arbitrary scalar factors. We now have two sets of four equations with four unknowns each. The two sets of unknowns are $a \lambda_{1} / \lambda_{5}, \cdots, d \lambda_{4} / \lambda_{5}$ and $a^{\prime} \lambda_{1} / \lambda_{6}, \cdots, d^{\prime} \lambda_{4} / \lambda_{6}$. The solutions are similar to eqs. (2):

$$
\begin{aligned}
a \frac{\lambda_{1}}{\lambda_{5}}=-\frac{M_{1}}{M_{5}}, & b \frac{\lambda_{2}}{\lambda_{5}}=\frac{M_{2}}{M_{5}}, & c \frac{\lambda_{3}}{\lambda_{5}}=-\frac{M_{3}}{M_{5}}, & d \frac{\lambda_{4}}{\lambda_{5}}=\frac{M_{4}}{M_{5}} \\
a^{\prime} \frac{\lambda_{1}}{\lambda_{6}}=-\frac{M_{1}^{\prime}}{M_{5}}, & b^{\prime} \frac{\lambda_{2}}{\lambda_{6}}=\frac{M_{2}^{\prime}}{M_{5}}, & c^{\prime} \frac{\lambda_{3}}{\lambda_{6}}=-\frac{M_{3}^{\prime}}{M_{5}}, & d^{\prime} \frac{\lambda_{4}}{\lambda_{6}}=\frac{M_{4}^{\prime}}{M_{5}}
\end{aligned}
$$

with $M_{i}^{\prime}$ denoting determinants in which $\mathbf{X}_{5}$ is replaced by $\mathbf{X}_{6}$. Unlike the affine case, these solutions are not invariant. However, we can find cross ratios of them which are absolute projective invariants, i.e. cross ratios that eliminate all the $\lambda_{i}$ :

$$
I_{1}=\frac{a b^{\prime}}{a^{\prime} b}=\frac{M_{1} M_{2}^{\prime}}{M_{1}^{\prime} M_{2}}, \quad I_{2}=\frac{a c^{\prime}}{a^{\prime} c}=\frac{M_{1} M_{3}^{\prime}}{M_{1}^{\prime} M_{3}}, \quad I_{3}=\frac{a d^{\prime}}{a^{\prime} d}=\frac{M_{1} M_{4}^{\prime}}{M_{1}^{\prime} M_{4}}
$$


We turn now to the $2 \mathrm{D}$ quantities. Our unknown quantities can be written in terms of 2D relative invariants, similarly to eqs. (3),(4),(5), with the first unknown being a free parameter $\mu$ :

$$
\begin{gathered}
a \frac{\lambda_{1}}{\lambda_{5}}=\mu \\
b \frac{\lambda_{2}}{\lambda_{5}}=\frac{1}{m_{15}}\left(m_{12}-\mu m_{25}\right) \\
c \frac{\lambda_{3}}{\lambda_{5}}=\frac{-1}{m_{15}}\left(m_{13}-\mu m_{35}\right) \\
d \frac{\lambda_{4}}{\lambda_{5}}=\frac{1}{m_{15}}\left(m_{14}-\mu m_{45}\right)
\end{gathered}
$$

Similar equations hold for $a^{\prime}, b^{\prime}, c^{\prime}, d^{\prime}$, with $\mu^{\prime}, m_{i j}^{\prime}, \lambda_{6}$ replacing $\mu, m_{i j}, \lambda_{5}$.

We can now eliminate the $\lambda_{i}$ on the left hand sides above using the same cross ratios as in the $3 \mathrm{D}$ case, eq. (14). We obtain

$$
\begin{aligned}
& I_{1}=\frac{a b^{\prime}}{a^{\prime} b}=\frac{\mu\left(m_{12}^{\prime}-\mu^{\prime} m_{25}\right)}{\mu^{\prime}\left(m_{12}-\mu m_{25}\right)} \\
& I_{2}=\frac{a c^{\prime}}{a^{\prime} c}=\frac{\mu\left(m_{13}^{\prime}-\mu^{\prime} m_{35}\right)}{\mu^{\prime}\left(m_{13}-\mu m_{35}\right)} \\
& I_{3}=\frac{a d^{\prime}}{a^{\prime} d}=\frac{\mu\left(m_{14}^{\prime}-\mu^{\prime} m_{45}\right)}{\mu^{\prime}\left(m_{14}-\mu m_{45}\right)}
\end{aligned}
$$

This is a simple quadric surface in invariant space, parametrized by $\mu, \mu^{\prime}$ :

$$
\begin{aligned}
& \mu m_{12}^{\prime}-I_{1} \mu^{\prime} m_{12}+\mu \mu^{\prime}\left(I_{1}-1\right) m_{25}=0 \\
& \mu m_{13}^{\prime}-I_{2} \mu^{\prime} m_{13}+\mu \mu^{\prime}\left(I_{2}-1\right) m_{35}=0 \\
& \mu m_{14}^{\prime}-I_{3} \mu^{\prime} m_{14}+\mu \mu^{\prime}\left(I_{3}-1\right) m_{45}=0
\end{aligned}
$$

It is easy to eliminate the terms proportional to $\mu, \mu^{\prime}$. The remaining (last above) equation is devided by $\mu \mu^{\prime} m_{12} m_{13}^{\prime} m_{14}^{\prime} m_{25}$. Defining now the $2 \mathrm{D}$ projective invariant cross ratios

$$
i_{1}=\frac{m_{12}^{\prime} m_{14}}{m_{12} m_{14}^{\prime}} \quad i_{2}=\frac{m_{12}^{\prime} m_{35}}{m_{13}^{\prime} m_{25}} \quad i_{3}=\frac{m_{12}^{\prime} m_{13}}{m_{12} m_{13}^{\prime}} \quad i_{4}=\frac{m_{12}^{\prime} m_{45}}{m_{14}^{\prime} m_{25}}
$$

we finally obtain:

Theorem 2. The $3 D$ absolute projective invariants of six generic points in $3 D$ are given in terms of $2 D$ invariants as

$I_{3}\left(I_{2}-1\right) i_{1} i_{2}-I_{3}\left(I_{1}-1\right) i_{1}-I_{1}\left(I_{2}-1\right) i_{2}=I_{2}\left(I_{3}-1\right) i_{3} i_{4}-I_{2}\left(I_{1}-1\right) i_{3}-I_{1}\left(I_{3}-1\right) i_{4}$ 


\section{Applications}

We will apply the above results to recognize 3D objects invariantly. For this purpose, we build a 3D invariant space in which recognition will take place. Each 3D 5-tuple is represented as a point in this space, with invariant coordinates $I_{1}, I_{2}, I_{3}$ (Fig 1). A 3D model is represented by a set of points in this space. (There is also some way of identifying these points as part of the same model.) The problem now is how to match the image to the model. We will distinguish several applications. They will be described using the affine approximation, but we will show later that the projective case needs only a small modification, requiring only 5 -tuples.

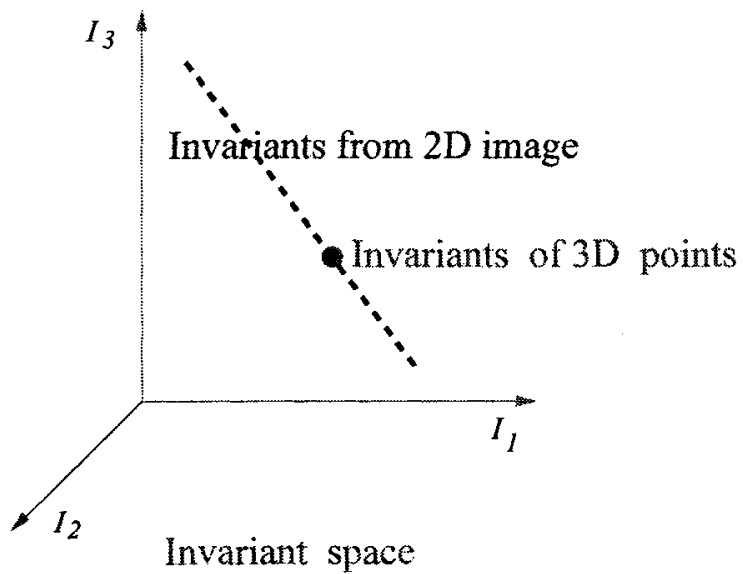

Fig. 1. Invariant space

1) Single image. Since the depth information is lost in this case, we cannot recognize an object without having a model. The simplest model can consist of five points in space, giving rise to three invariants $I_{1}, I_{2}, I_{3}$. These can be represented as a point in the 3D invariant space. (Of course a realistic model will consist of many such points). To recognize the model in the image, we extract 5 -tuples of features from the image and calculate their $2 \mathrm{D}$ invariants $i_{\alpha \beta}$. Using Theorem 1 (or 2), we have two equations relating the three 3D invariants $I_{1}, I_{2}, I_{3}$. Geometrically, we obtain a space line in our $3 \mathrm{D}$ invariant space. If a 5 -tuple in the $2 \mathrm{D}$ image is a projection of some 5-tuple in 3D, then the line obtained from this 2D 5-tuple will pass through the point in invariant space representing the 3D 5-tuple (Fig. 1). That is, we have found the correspondence between the 2D and 3D 5-tuples. A different view of the 5-tuple will give rise to a different line in 3D, but still passing through the same point. To recognize objects we thus look for instances in which lines obtained from the image pass through points representing models in the invariant 3D space. 
2) Multiple images. Although we concentrate on single views, multiple view applications are also valuable. For instance it helps in the symmetry case (described next) which can be regarded as multiple views. Here we do not need a model to recover the depth information, only for the identification. We use our method to find correspondences between the images. We first extract a 5-tuple of features from one of the images and transform it into a line in the 3D invariant space.

Next, we look at a different image, in which objects are seen from a different viewpoint. Again we draw a line in the same 3D space of invariants as before. If this line meets the first line in 3D then the two 5-tuples have the same 3D invariants $I_{i}$. These are the coordinates of the intersection point. This means that the two 5-tuples are affine equivalent. (We later generalize to the projective case). This in turn indicates that we may have two different views of the same 5-tuple. Again, we have found a correspondence between 5 -tuples, this time between two views.

With $n$ 5-tuples, the total number of lines in the invariant space is $O(n)$. We can find line intersections in a way similar to that used in Hough space, namely divide the space into bins and see if a certain bin has more than one line going through it. We do not need to check all bins; we only need to go along the known lines. A hierarchical scale space approach can be used to make the process more efficient. This brings our total complexity closer to $O(n)$ rather than $O\left(n^{2}\right)$ as in previous methods.

Based on the above discussion, our recognition algorithm involves the following steps:

i) Feature extraction. Find candidate 5-tuples that are potentially affine (or projective) equivalent. Various clues can be used to prune unpromising sets, e.g. non-matching links that are visible between the points, or parallelism.

ii) Invariant description. For each 5 -tuple that remains from (1), calculate the two equations for the three 3D invariants, namely plot a line in a $3 \mathrm{D}$ invariant space. Find all lines that meet in 3D. Each intersection represents the 3D affine invariants of two affine equivalent 5 -tuples. An object will be represented by several such intersection points.

iii) Recognition. We now have invariant 3D point sets representing various visible objects. Similarly, the models in the database can be represented by point sets in the same invariant 3D space. We can use several 5-tuples in each model, obtaining several points representing the model in the invariant space. Identification is now straightforward. If an object's point set falls on a model's point set in the invariant space, than the object is identified with the model. No search is needed for the right model, because the invariant point sets of the models can be indexed according to their coordinates. No search is needed for the viewpoint or pose either because of the invariance. As many points as practical need to be used to increase reliability.

iv) Verification. This step is independent of the invariants method. It overcomes any errors that we may have in calculating the determinants $I_{i}$. Using the 2D coordinates of the images, and the correspondence found in (ii), we calcu- 
late the 3D coordinates of the features. Now we can find the 3D transformation (pose) that produces the best fit between the 3D object and the model identified in (iii), using least squares fitting. The identification is rejected if the fitting error is too big. We may try to fit several models to each object to find the best fit.

3) Single image, symmetric models. The problem encountered earlier in the single-image case was the unknown parameter along the space line, resulting from the missing depth information. Instead of using a model as was done in case (1), we can use a modeling assumption. One modeling assumption that we use is symmetry. Most man-made objects are symmetric, e.g. vehicles, tanks, airplanes and buildings. Symmetry is also found in human and animal bodies. The symmetry is observed explicitly only in a frontal view. In any other view the symmetry is observed as a skew-symmetry. Many researchers have used skewsymmetry for recognition, but with serious limitations. They usually assume that the skew-symmetry itself is known, i.e. we know which feature in one half of the object corresponds to which feature in the symmetric half. In other words, they assume that the correspondence problem has already been solved. Here we make no such assumption but detect the skew-symmetric objects in an image.

The two halves of a skew-symmetric object are affine equivalent. Therefore we can apply the algorithm described above which was designed to find affine equivalent 5 -tuples. Having found matching 5 -tuples, we have to verify that they are halves of the same object. The lines connecting corresponding points in a symmetric object are parallel in $3 \mathrm{D}$, therefore they will be parallel in an affine projection, and this is easy to check.

The verification step (iv) is easier because of the symmetry assumption. The skew-symmetric object that we have found can be rectified, using an affine transformation, to obtain a standard view in which the object is (non-skew) symmetric. It can then be matched directly with a database of symmetric models.

\section{Implementation Issues}

Experiments performed so far with the method seem very encouraging. We have used only a small number of models, but the robustness to errors seems quite good, so that we can safely add more models.

During the implementation, a number of problems had to be overcome. We briefly summarize some of them here, along with their solutions.

1) Low level image processing. It is not the goal of this research to develop new methods of feature extraction. Rather, we use the model-based approach to overcome the problems inherent in feature detection. Nevertheless, the inadequacies of feature detection are so great that we must do some preprocessing before using our method. The positions of feature points are very inaccurate, so we have concentrated on finding lines and their intersections rather than points. However, we usually have far too many lines, most of which are irrelevant to the object (Fig. 2). 
Solution: We keep only those lines that lie along principal directions. Most man-made objects such as vehicles and buildings have a small number of directions, e.g. major axes, to which most prominent lines are parallel. These are relatively easy to find. Fig. 3 (left) shows the lines left after the above pruning.
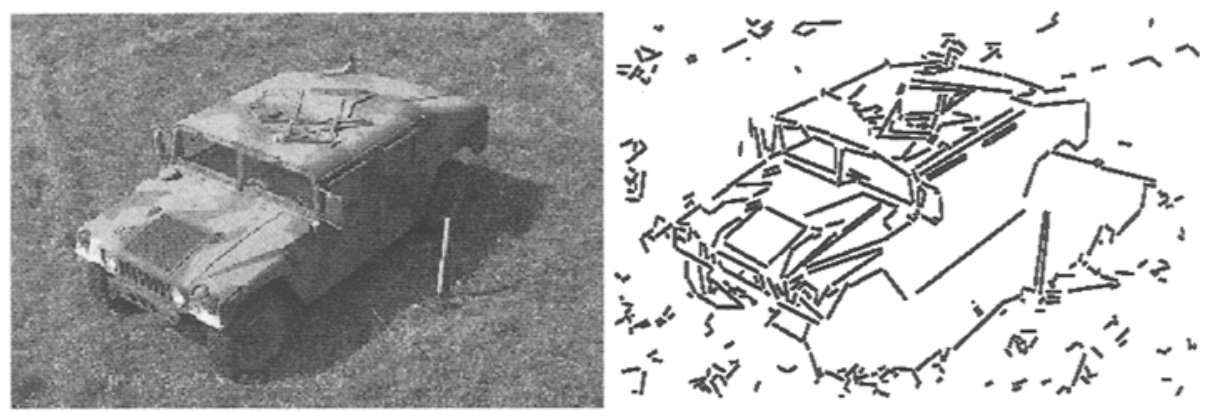

Fig. 2. Image and detected lines
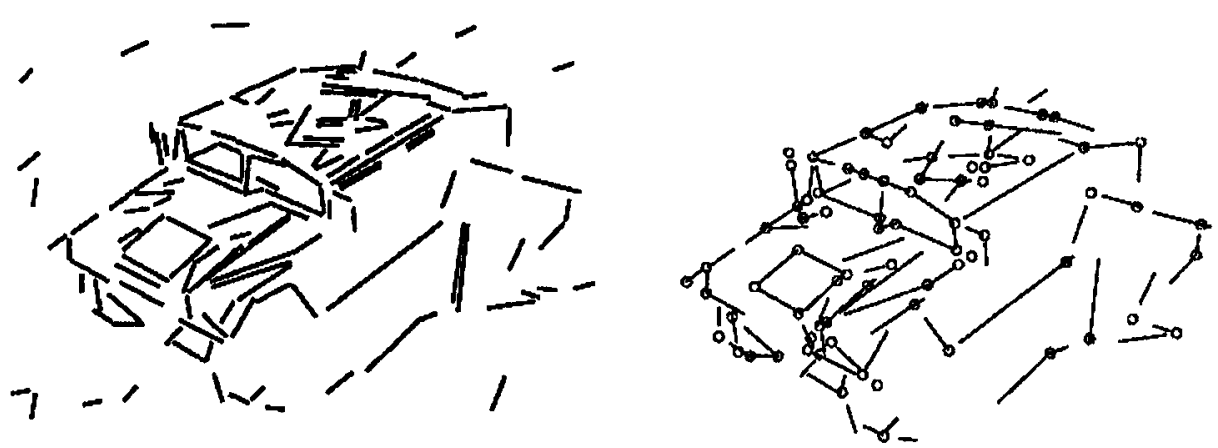

Fig. 3. Lines along principal directions (left), corner features (right).

2) Large numbers of 5-tuples. With the number of features in the hundreds, the number of all possible quintuples is on the order of $100^{5}$, which is quite prohibitive.

Solution: We use only connected quintuples, i.e. 5-tuples whose member points are connected to each other by visible lines. Four points may be connected to form a (3D) corner, with one central point connected to three others, and the connections lying along principal directions. The fifth point can be any 
point connected to these four. Thus the number of possible quintuples (and invariants) is reduced to on the order of $10^{3}$. Fig. 3 (right) shows the resulting corners.

3) Perspectivity. The affine (orthographic) approximation that we initially assumed was found to be inadequate in many cases. We had to use the full perspective treatment. However, this normally requires 7-point sets rather than the 5-point sets we have been using, which increases complexity significantly.

Solution: We use vanishing points, namely the points at which the lines along a principal direction intersect in the image. This is based on a (quite common) assumption that when several lines intersect in an image, they probably intersect in 3D as well. Thus we have obtained some known correspondences between 2D and 3D points without increasing complexity. The invariant calculation is quite insensitive to the exact location of the vanishing points. Fig. 4 illustrates the vanishing point method.

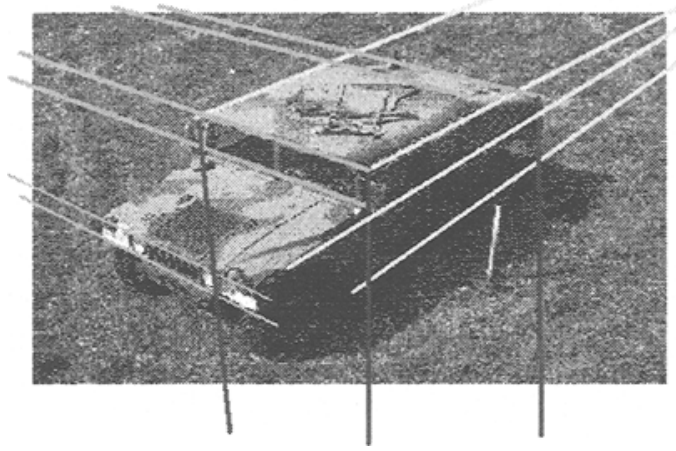

Fig. 4. Vanishing points

4) Finding intersections. Finding the point-line intersections in the 3D invariant space needs to be done efficiently, with low complexity. Given a line, we want to search for intersecting points only in the neighborhood of this line. For this purpose we can borrow methods from GIS (Geographic Information Systems). These types of problems have been solved there in the 2D case using quadtrees. We can extend these methods to 3D using octrees.

\section{Experimental Results}

\subsection{Single Views}

We show here results obtained from two different images of a HMMWV vehicle.

The first step is building a 3D model in the invariant space described earlier. We start from a "real" 3D model of a vehicle (downloaded from the Internet; 
Fig. 5), and choose a "base" of four corner points on it. These can be assigned the coordinates of a standard affine coordinate base, namely

$$
\mathbf{X}_{1}=(1,0,0,1), \mathbf{X}_{2}=(0,1,0,1), \mathbf{X}_{3}=(0,0,1,1), \mathbf{X}_{4}=(0,0,0,1)
$$

The original model can be transformed by a unique affine transformation so that the four base points will take on the above values. This has no effect on our treatment since it is invariant. Doing that, any fifth point of the model is transformed to a point with some coordinates $\mathbf{X}=(X, Y, Z, 1)$.
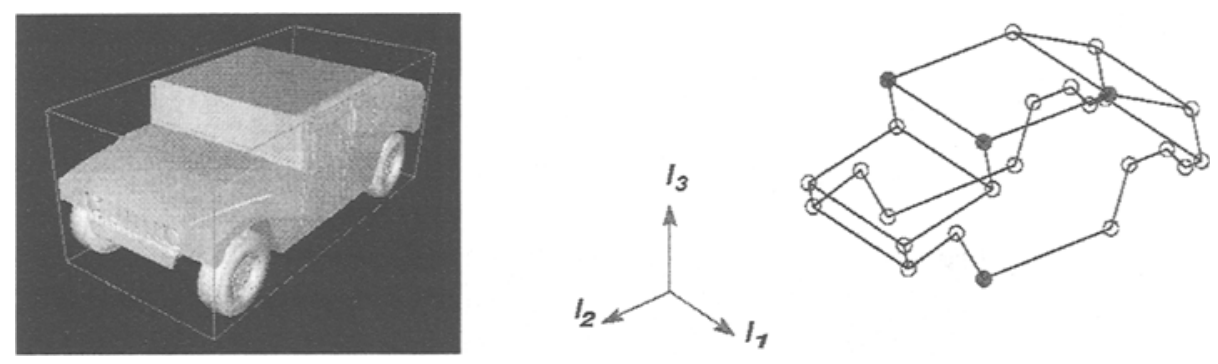

Fig. 5. Original and invariant models

It is easy to see that these new coordinates are in fact invariants. One way is to explicitly calculate the invariants as given earlier, to obtain

$$
I_{1}=X, \quad I_{2}=Y, \quad I_{3}=Z
$$

Another way is to note that such a base is a standard, or canonical base that can always be obtained from any given affine transformed version of the model. That makes it an invariant base and any quantity expressed with respect to it is thus invariant.

The model we thus obtain in invariant space is shown in Fig 5 (right).

Next, given one view of the vehicle, we calculate the lines in 3D invariant space according to Theorem 2. The results for each view are shown in Fig 6 .

An intersection of a line with a point in 3D means that a correspondence has been found between the 5-point set in the image, which gave rise to the line, and a 5-point set in the 3D model that gave rise to the point. In theory, we need only $6-7$ feature correspondences. This is enough to calculate the pose (or camera coordinates). We can then project the model on the image and do a more detailed match. However, more intersections give higher reliability.

Reliability: We have obtained rather good results for the intersections (Fig. 6). Out of 13 lines that were supposed to intersect, 10 intersected within the error circles marked in the figure, two missed and one was a "coincidental" intersection. We have introduced an artificial error of $10 \%$ in the positions of the feature points, and have still obtained an acceptable number of 7-8 intersections. 

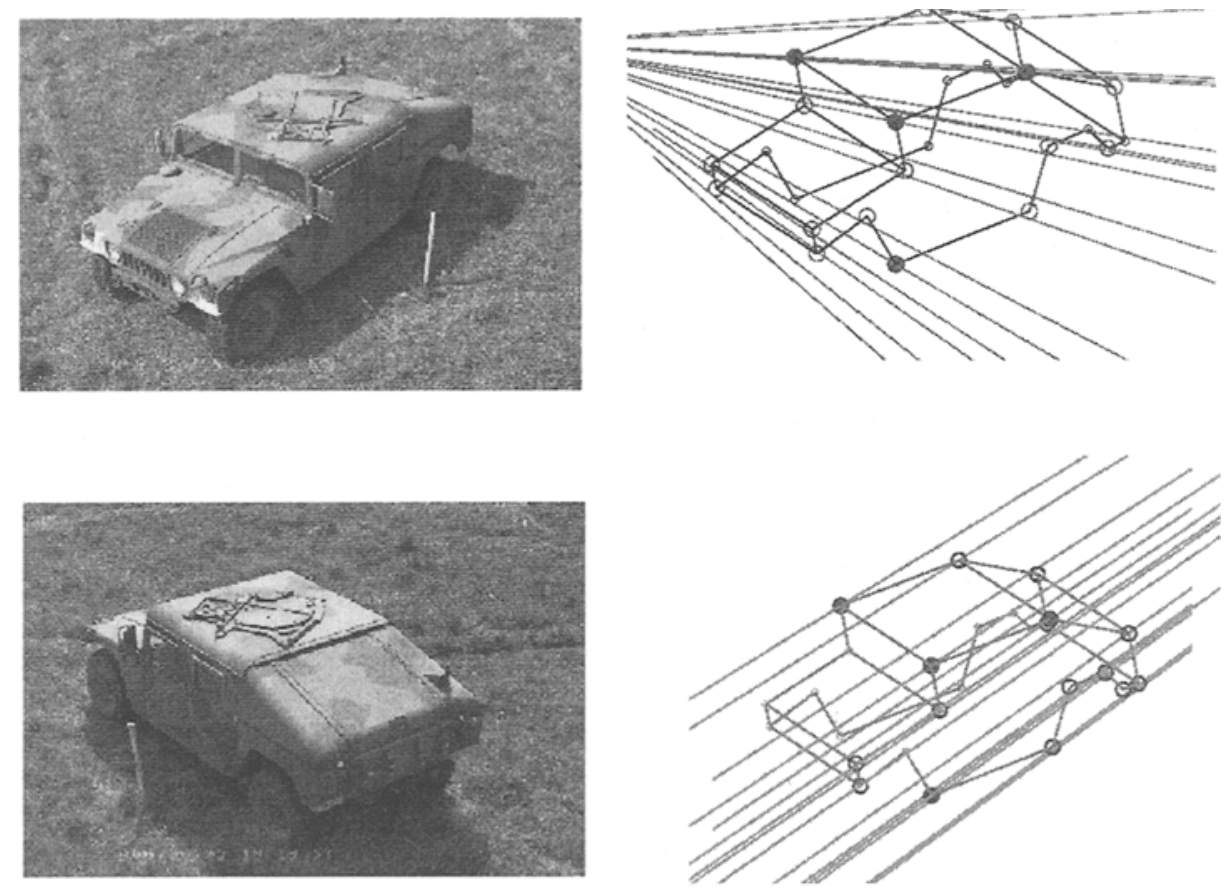

Fig. 6. Intersections in invariant space between image lines and model points, for each view.

The lines converge to a point representing the position of the camera center in invariant space, making it easy to calculate the pose. Thus the lines are in fact a representation of the light rays in invariant space.

\subsection{Two Views}

We have applied the method described above to find a correspondence between two views, without a model. Applications include: (1) "stereo" with widely differing images, such as the two views above; (ii) $3 \mathrm{D}$ model construction from $2 \mathrm{D}$ images.

The correspondences are found by drawing the lines in 3D invariant space generated by each image, and then finding the intersections between the space lines. A line intersection indicates that there is a correspondence between the appropriate 5-point sets from the two images. The intersection point is also a point of the unknown invariant 3D model. 


\section{Summary}

We summarize our object recognition method as follows.

- Build a model in invariant 3D space.

- From 5-tuples in the image, draw lines in 3D space.

- Find intersections of the above lines with model points, tentatively identifying one or a few models.

- Find the pose and project the tentative model(s) on the image.

- Perform final matching and identification between the projected model(s) and the image.

\section{References}

1. E. Barrett. Personal communication.

2. P. Beardsley, P. Torr, and A. Zisserman. 3D Model Aquisition from Extended Image Sequences. Proc. European Conf. on Computer Vision, 683-695, 1996.

3. J. Ben-Arie, Z. Wang, and R. Rao. Iconic Recognition with Affine-Invariant Spectral Signatures. Proc. ICPR A, 672-676, 1996.

4. T.O. Binford and T.S. Levitt. Model-Based Recognition of Objects in Complex Scenes. Proc. DARPA Image Understanding Workshop, 89-100, 1996.

5. A. Bruckstein, E. Rivlin, and I. Weiss. Scale Space Invariants for Recognition. Machine Vision and Applications, 15:335-344, 1997.

6. J.B. Burns, R. Weiss, and E.M. Riseman. View Variation of Point Set and Line Segment Features. Proc. DARPA Image Understanding Workshop, 650-659, 1990.

7. S. Carlsson. Relative Positioning from Model Indexing, Image Vision Comut., 12:179-186, 1994.

8. R.W. Curwen and J.L. Mundy. Grouping Planar Projective Symmetries. Proc. DARPA Image Understanding Workshop, 595-605, 1997.

9. R. Deriche, Z. Zhang, Q.T. Luong, and O.D. Faugeras. Robust Recovery of the Epipolar Geometry for an Uncalibrated Stereo Rig. Proc. European Conf. on Computer Vision, A567-576, 1994.

10. H. Guggenheimer. Differential Geometry. Dover, New York, 1963.

11. J.P. Hopcroft, D.P. Huttenlocher, and P.C. Wayner. Affine Invariants for ModelBased Recognition. In Geometric Invariance in Machine Vision, eds. J.L. Mundy and A. Zisserman, MIT Press, Cambridge, MA, 1992.

12. D. Jacobs. Space Efficient 3D Model Indexing. Proc. IEEE Conf. on Computer Vision and Pattern Recognition, 439-444, 1992.

13. D. Jacobs and R. Basri. 3-D to 2-D Recognition with Regions. Proc. IEEE Conf. on Computer Vision and Pattern Recognition, 547-553, 1997.

14. D. Keren, R. Rivlin, I. Shimshoni, and I. Weiss. Recognizing 3D Objects using Tactile Sensing and Curve Invariants. University of Maryland Technical Report CS-TR-3812, 1997.

15. S.J. Maybank. Relation between 3D Invariants and 2D Invariants. Image Vision Comut., 16:13-20, 1998.

16. P. Meer and I. Weiss. Point/Line Correspondence under Projective Transformations. Proc. ICPR A, 399-402, 1992. 
17. R. Mohr, L. Quan, and F. Veillon. Relative 3D Reconstruction using Multiple Uncalibrated Images. Intl. J. Robotics Research, 14:619-632, 1995.

18. S. Peleg, A. Shashua, D. Weinshall, M. Werman, and M. Irani. Multi-Sensor Representation of Extended Scenes using Multi-View Geometry. Proc. DARPA Image Understanding Workshop, 79-83, 1997.

19. E. Rivlin and I. Weiss. Local Invariants for Recognition. IEEE Transactions on Pattern Analysis and Machine Intelligence, 16:226-238, 1995.

20. E. Rivlin and I. Weiss. Recognizing Objects Using Deformation Invariants. Computer Vision and Image Understanding, 65:95-108, 1997.

21. C.A. Rothwell. Object Recognition through Invariant Indexing. Oxford University Press, Oxford, 1995.

22. A. Shashua and N. Navab. Relative Affine Structure: Canonical Model for 3D from 2D Geometry and Applications. IEEE Transactions on Pattern Analysis and Machine Intelligence, 18:873-883, 1996.

23. J. Sato and R. Cipolla. Affine Integral Invariants for Extracting Symmetry Axes. Image and Vision Computing, 15:627-635, 1997.

24. G. Sparr. Projective Invariants for Affine Shapes of Point Configurations. Proc. 1st Workshop on Invariance, 151-170, 1991.

25. C.E. Springer. Geometry and Analysis of Projective Spaces. Freeman, San Francisco, 1994.

26. P.F. Stiller, C.A. Asmuth, and C.S. Wan. Invariant Indexing and Single View Recognition. Proc. DARPA Image Understanding Workshop, 1423-1428, 1994.

27. B. Strumpel. Algorithms in Invariant Theory. Springer Verlag, New York, 1993.

28. L. Van Gool, T. Moons, E. Pauwels, and A. Oosterlinck. Vision and Lie's Approach to Invariance. Image and Vision Computing, 13:259-277, 1995.

29. T. Vieville, O. Faugeras, and Q.T. Luong. Motion of Points and Lines in the Uncalibrated Case. Intl. J. Computer Vision, 17:7-41, 1996.

30. B. Vijayakumar, D.J. Kriegman, and J. Ponce. Structure and Motion of Curved 3D Objects from Monocular Silhouettes. Proc. IEEE Conf. on Computer Vision and Pattern Recognition, 327-334, 1996.

31. I. Weiss. Geometric Invariants of Shapes. Proc. DARPA Image Understanding Workshop, 1125-1134, 1988.

32. I. Weiss. Noise Resistant Invariants of Curves. IEEE Transactions on Pattern Analysis and Machine Intelligence, 15:943-948, 1993.

33. I. Weiss. Geometric Invariants and Object Recognition. Intl. J. Computer Vision, 10:207-231, 1993.

34. I. Weiss. Local Projective and Affine Invariants. Annals of Mathematics and Artificial Intelligence, 13:203-225, 1995.

35. I. Weiss. 3D Curve Reconstruction from Uncalibrated Cameras. University of Maryland CS-TR-3605. Also in Proc. ICPR A, 323-327, 1996.

36. I. Weiss. Model-based Recognition of 3D Curves from One View. Journal of Mathematical Imaging and Vision, in press, 1997.

37. M. Zerroug and R. Nevatia. Using Invariance and Quasi-invariance for the Segmentation and Recovery of Curved Objects. In Lecture Notes in Computer Science 825, Springer-Verlag, Berlin, 1994.

38. A. Zisserman, D.A. Forsyth, J.L. Mundy, C.A. Rothwell, J. Liu, and N. Pillow. 3D Object Recognition using Invariance. Artificial Intelligence 78:239-288, 1995. 